3 Harrington MG, Macpherson P, Mcintosh WB, Allam BF, Bone I. The significance of the incidental finding of basal ganglia calcification on computerised tomography. f Neurol Neurosurg Psychiatry 1981;44: 1168-70.

4 Lowenthal A, Bruyn GW. Calcification of the striatopallido dentate system. In: Bruyn GW, Vinken P, eds. Handbook of neurology. Vol 6. Vinken P, eds. Handbook of neurology. Vol 6.
Amsterdam: North Holland, 1968:703-25.

5 Cohen CR, Duchesnau PM, Weinstein MA Calcification of the basal ganglia as visualised by computerised tomography. Radiology
1980;134:97-9.

A sporadic case of dentatorubral pallidoluysian atrophy (DRPLA) with CAG repeat expansion but no clinical abnormalities in the father

Dentatorubral pallidoluysian atrophy (DRPLA) is an autosomal dominant neurodegenerative disorder characterised by various combinations of myoclonus, epilepsy, ataxia, choreoathetosis, and dementia. ${ }^{1}$ Neuropathological changes consist of combined degeneration of the dentatorubral and pallidoluysian systems of the brain. The disease predominantly occurs as an inherited condition and is more common in Japan than in other countries. ${ }^{12}$ Unstable expansion of a CAG trinucleotide repeat in a gene on the short arm of chromosome 12 was recently identified as the pathogenic mutation in DRPLA. ${ }^{34}$ There is a good correlation between the clinical signs of DRPLA and CAG repeat size. Patients with earlier onset tend to have a phenotype of progressive myoclonus epilepsy and larger expansions. ${ }^{3-6}$ Whereas the penetrance of DRPLA in Japan is estimated to be high $(90 \%),{ }^{7}$ sporadic patients with expanded alleles have been known. ${ }^{5}$ However, to the best of our knowledge, DNA analysis of the DRPLA gene of both parents of a sporadic patient has never been documented. In the present paper, we report a sporadic case of DRPLA and the results of analysis of the DRPLA gene in the patient and her parents.

A 39 year old woman had been well until the age of 27 , when she started to experience generalised epileptic seizures, slurred speech, unsteady gait, and intellectual disturbances. All these symptoms were slowly progressive. At the age of 39 she complained of increased frequency of the epileptic attacks and progressive intellectual and motor dysfunction. Her medical history was unremarkable. Her family history disclosed no members with ataxia, epilepsy, involuntary movements, psychosis, or other neurodegenerative diseases (figure). Neither her 66 year old father nor her 63 year old mother had any neurological abnormalities or epilepsy. Her three children and her 35 year old sister were also free of neurological symptoms.

Examination of the patient showed that she was awake and had pronounced dementia. Her IQ on the Tanaka-Binet scale was 16 , which corresponds to the intelligence of a child aged 2 years 11 months. Her speech was slurred and explosive. External ocular movements were full in range, and there was no nystagmus. Other cranial nerves were intact. The patient exhibited facial grimacing and choreoathetoid-like movements of her hands and feet. No myoclonus or tremors were noted. There was no motor weakness or amyotrophy. All four limbs were hypotonic. The patient exhibited ataxia in all four limbs and the trunk. Her gait was wide based and very ataxic. Babinski's sign was present bilaterally, but deep tendon

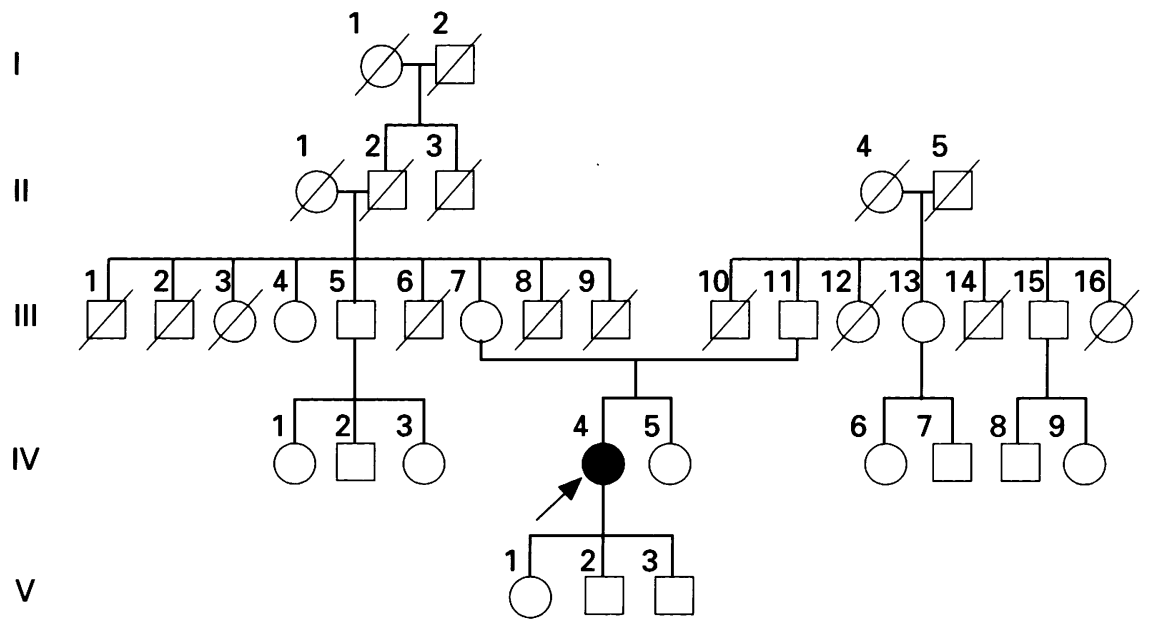

Pedigree of the patient. The proband is indicated by the arrow. Closed symbol indicates affected member, open symbols, unaffected members; circles, females, squares, males; diagonal lines, deceased persons. II-3, poor school performance; II-4, cerebrovascular disease, died at 46 years; II-5, colon cancer, died at 74 years; III-1, died before 1 year; III-2, poor school performance, died at 47 years; III-3, died before 1 year; III-4, 70 years, chronic renal failure; III-5, 68 years, chronic renal failure; III-6, killed in the war at 19 years, III-7, 63 years, patient's mother; III-8, poor school performance, cerebral haemorrhage, died at 32 years; III-9, died at 3 years; III-10, died at 5 years; III-11, 66 years, patient's father; III-12, died at 2-3 years; III-13, 60 years; III-14, died at 5-6 years; III-15, died at 58 years; III-16, died at 1-2 years; IV-1, 40 years, diabetes mellitus; IV-4, the proband.

reflexes were normal. There were no abnormalities of the sensory or autonomic nervous system. Brain MRI showed moderate atrophy of the cerebellar vermis and hemispheres, the brainstem, more prominently in the tegmentum, and the cerebrum. An EEG showed diffuse, slow basic activity, a few $2-3 \mathrm{~Hz}$ waves, single spikes, and polyspikes. High frequency photic stimulation induced photomyoclonus. The patient's symptoms progressed and she became bedridden and mute over the next few months.

DNA analysis of the DRPLA gene of leucocytes was performed after informed consent had been obtained. The method of polymerase chain reaction (PCR) amplification and method of analysing the CAG repeat in the DRPLA gene have been described previously. ${ }^{3}$ The CAG repeat numbers were $64 / 15$ in the patient, $59 / 18$ in her 66 year old father, and $17 / 18$ in her 63 year old mother.

The present patient apparently represents a sporadic case of hereditary DRPLA. This report is, to the best of our knowledge, the first documentation of DNA analysis of the DRPLA gene in parents and a child in a sporadic case. Although an asymptomatic father aged 54 years with an expanded CAG repeat was reported by Komure et al, ${ }^{6}$ there is no mention of DNA analysis of the mother. This family is interesting and important with regard to how DRPLA may arise.

The number of CAG repeats on the DRPLA gene ranges from 45 to 88 in patients with DRPLA ${ }^{3-6}$ and from 7 to 35 in normal subjects. ${ }^{3-68}$ There is no overlap between the distribution of the number of CAG repeats in normal subjects and patients. The age of onset of DRPLA diagnosed by both clinical features and DNA analysis is reported to range from 4 to 62 years, ${ }^{5}$ and an inverse correlation between numbers of CAG repeat units and age at onset has been shown. ${ }^{3-6}$ This inverse correlation seemed to be less strong in an adult onset case than an early onset case. ${ }^{3-6}$ Thus persons who have a mild expansion of CAG repeats may not develop clinical symptoms by their $60 \mathrm{~s}$, as in the father of this patient. Those with 59 CAG repeats would be expected to experience the onset of clinical symptoms in their $50 \mathrm{~s}$ based on the results of a correlation analysis. ${ }^{5}$ The age of 66 is beyond the range of ages of onset of DRPLA hitherto documented. Nevertheless, all evidence would indicate that the father is at high risk.

There are three possible mechanisms to explain how the trinucleotide repeat length expanded: firstly, expansion via intermediate size alleles, secondly, via asymptomatic fully expanded alleles, and thirdly, de novo mutation from normal alleles. Our case is thought to represent the second mechanism. The distribution of repeat numbers in the DRPLA gene in the normal Japanese population has been reported, and $7 \cdot 4 \%$ of the alleles in Japan have greater than 19 repeats. ${ }^{8}$ By contrast, none of the alleles in white people and only $1 \%$ of the alleles in Afro-Americans are of this size. ${ }^{8}$ In addition, two intermediate size alleles with 32 and 34 repeats have been reported in normal Japanese. ${ }^{4}$ Thus Burke et al suspect the expansion via intermediate size alleles to fully expanded alleles in succeeding generations for Japanese DRPLA. ${ }^{8}$ However, this first mechanism, expansion via intermediate size alleles, has never been found in a sporadic case of DRPLA, although it has been shown in sporadic cases of Huntington's disease. ${ }^{9}$

Thus a person who shows clinical features resembling those of hereditary diseases without family history, represents the first family member to cross the phenotypic threshold, which is a product of CAG repeat length, time, and other unknown features that govern its onset. The number of CAG repeats in this family increased from 59 to 64 as a result of paternal transmission. In triplet repeat disease caused by expanded CAG repeats, it should be noted that intergenerational increases in CAG repeats in paternal transmission are the major source of the larger expansions.

This work was supported in part by a grant in aid for Scientific Research on Priority Areas and a grant in aid for Creative Basic Research (Human Genome) from the Ministry of Education, Science, Sports and Culture of Japan, a Grant from the Research Committee for Ataxic Diseases of the 
Ministry of Health and Welfare of Japan, and special coordination funds from the Japanese Science and Technology Agency.

N SHIMIZU T YAMAMI M NAKAYAMA Department of Neurology, Teikyo University School of Medicine, Ichihara Hospital, Ichihara, fapan T IKEUCHI R KOIDE S TSUJI Department of Neurology, Brain Research Institute, Niigata University, Niigata, Fapan

Correspondence to: Dr Natsue Shimizu, Department of Neurology, Teikyo University School of Medicine, Ichihara Hospital, 3426-3 Anesaki, Ichihara-shi, Chiba-ken, Japan 299-04.

1 Naito H, Oyanagi S. Familial myoclonus epilepsy and choreoathetosis: hereditary dentatorubral-pallidoluysian atrophy. Neurology

2 Takahashi H, Ohama E, Naito $\mathrm{H}$, et al. Hereditary dentatorubral-pallidoluysian atrophy: Clinical and pathologic variants in a family. Neurology 1988;38:1065-70.

3 Koide $\mathrm{R}$, Ikeuchi $\mathrm{T}$, Onodera $\mathrm{O}$, et al. Unstable expansion of CAG repeat in hereditary dentatorubral-pallidoluysian atrophy (DRPLA). Nature Genetics 1994;6:9-13.

4 Nagafuchi S, Yanagisawa H, Sato K, et al. Dentatorubral and pallidoluysian atrophy expansion of an unstable CAG trinucleotide on chromosome 12p. Nature Genetics 1994;6: on -8 .

5 Ikeuchi $\mathrm{T}$, Koide $\mathrm{R}$, Onodera $\mathrm{O}$, et al. Dentatorubral-pallidoluysian atrophy (DRPLA): clinical features are closely related to unstable expansions of trinucleotide
(CAG) repeat. Ann Neurol 1995;37:769-75.

6 Komure O, Sano A, Nishino N, ct al. DNA analysis in hereditary dentatorubral-pallianalysis in hereditary dentatorubral-palli-
doluysian atrophy: correlation between CAG repeat length and phenotypic variation and the molecular basis of anticipation. Neurology 1995;45:143-9.

7 Kondo K, Naito H, Oyanagi S. Heredity of dentatorubro-pallidoluysian atrophy. Annual Reports of the Research Committee for Neurological Degenerative Diseases 1981:222-6.

8 Burke JR, Ikeuchi T, Koide R, et al. Dentatorubral-pallidoluysian atrophy and Haw River syndrome. Lancet 1994;344:1711-2.

9 Goldberg YP, Kremer B, Andrew SE, et al. Molecular analysis of new mutations for Molecular analysis of new mutations for
Huntington's disease: intermediate alleles Huntington's disease: intermediate alleles
and sex of origin effects. Nature Genetics and sex of origi $1993 ; 5: 174-9$.

\section{Malignant distress on eye contact after severe head injury}

We report a patient with severe head injury who developed a very specific pattern of symptoms of distress on eye contact. It is likely that this neurobehavioural syndrome contributed to the decline of his mental and physical state which started three years after injury.

The patient was described as having been a quiet, private person who enjoyed solitary pursuits. He disliked being in the company of people who were disabled. At the age of 32 he had a severe closed head injury. Brain CT showed left temporal lobe contusions and an extradural haematoma at the base of skull, which was evacuated. $\mathrm{He}$ was in a coma for about four months. Two years after injury he was underweight and had severe contractures with no functionally useful movement.

At his best, about three years after injury, he was able to walk with the aid of a frame and assistance and was beginning to be able to feed himself. His most reliable cognitive response was following basic commands. At times he was able to indicate "yes" and "no", and name objects and colours, but this was mostly unreliable. He had severe dysarthria.
His best verbal output consisted of singing a few words of a well known song. He had never, since the accident, shown any definite recognition of anybody, including his wife.

About two to three years after injury he began to show increasing signs of tension and irritability during therapy. It seemed as though he wanted to be left alone. It was noted that "eye contact was reported to provoke anger-noise".

Since that time he has continued to show a behavioural syndrome consisting of symptoms of distress and agitation in relation to eye contact. Other social cues-for example, entering his room but avoiding eye contact-also cause distress but to a much lesser degree.

Symptoms of distress include wailing, grimacing, opisthotonic posturing, and repeated hitting of limbs, with facial engorgement and profuse sweating. These symptoms last for as long as the observer's gaze is maintained. The symptoms subside within a few seconds of disengagement from eye contact. All three authors, as well as several nurses involved in his care, have independently noted the ability of eye contact to provoke distress. Nursing staff have found that averting their eyes from his gaze reduces distress. His eye movements lack normal fluency. He tends to fixate on objects, particularly faces and eyes, and has difficulty directing his gaze away. As a consequence eye contact tends to be maintained, with a fixed unblinking stare, until the observer looks away.

Specific behaviour therapy aimed at increasing exposure to eye contact was unsuccessful. The symptom was associated with a steady decline in his mental and physical state such that by 1994, seven years after injury, he had to be nursed continuously on his bed. His weight dropped $20 \mathrm{~kg}$ to $51 \mathrm{~kg}$. Brain CT in January 1991 showed gross hydrocephalus with periventricular lucency but insertion of a ventriculoperitoneal CSF shunt at that time had no effect on his mental state. Over the past year combined treatment with trifluoperazine and moclobemide have been associated with a slight improvement in his mental and physical state.
To test the hypothesis that the distress was indeed related specifically to eye contact, rather than some other aspect of a person's face or simply the presence of human contact, we carried out the following brief experiment. In a state of rest, lying awake in his recliner with his eyes open, the patient was approached by an unfamiliar member of staff who stood almost facing him about three feet away. In one condition the staff member looked directly at the patient, and thereby invariably made eye contact; in the other condition the staff member faced the patient at the same angle, but with eyes averted by about $30^{\prime \prime}$ from the patient. An observer, hidden from view, noted the time taken to become agitated (defined as banging his arm on the chair). The rate of eye blinking during the two conditions was also noted. The testing condition was terminated by the person walking away as soon as the patient became agitated, or after 10 minutes if there was no sign of agitation by this time. A total of 12 sessions were studied, six of each condition, carried out on three separate days. The order of testing was counter balanced.

The mean time to agitation was very significantly shorter in the eye contact condition than in the eyes averted condition (mean 93 (SD 114) s v 510 (SD 221) s; P $=0.006$; figure). The rate of eye blink was significantly slower in the eye contact condition $(0.67$ (SD 0.78$) \quad v \quad 1.87$ (SD 0.42$)$ blinks $/ \mathrm{min} ; \mathrm{P}=0.034$ )

Our patient showed two problems. One was distress on eye contact. The other was an apparent inability to disengage his eyes from eye contact.

It is known that eye contact may be associated with arousal and this link seems to develop early in life. In animals directed gaze at another may be used as a form of threat (Rudyard Kipling's Mowgli “discovered that if he stared hard at any wolf, the wolf would look away"). The tendency to gaze aversion in monkeys develops during the first weeks of life. ${ }^{1}$ Cells in the superior temporal sulcus of the monkey are specifically tuned to detect eye contact or averted gaze. ${ }^{2}$ In humans various studies have shown that eye contact increases arousal. Hutt and

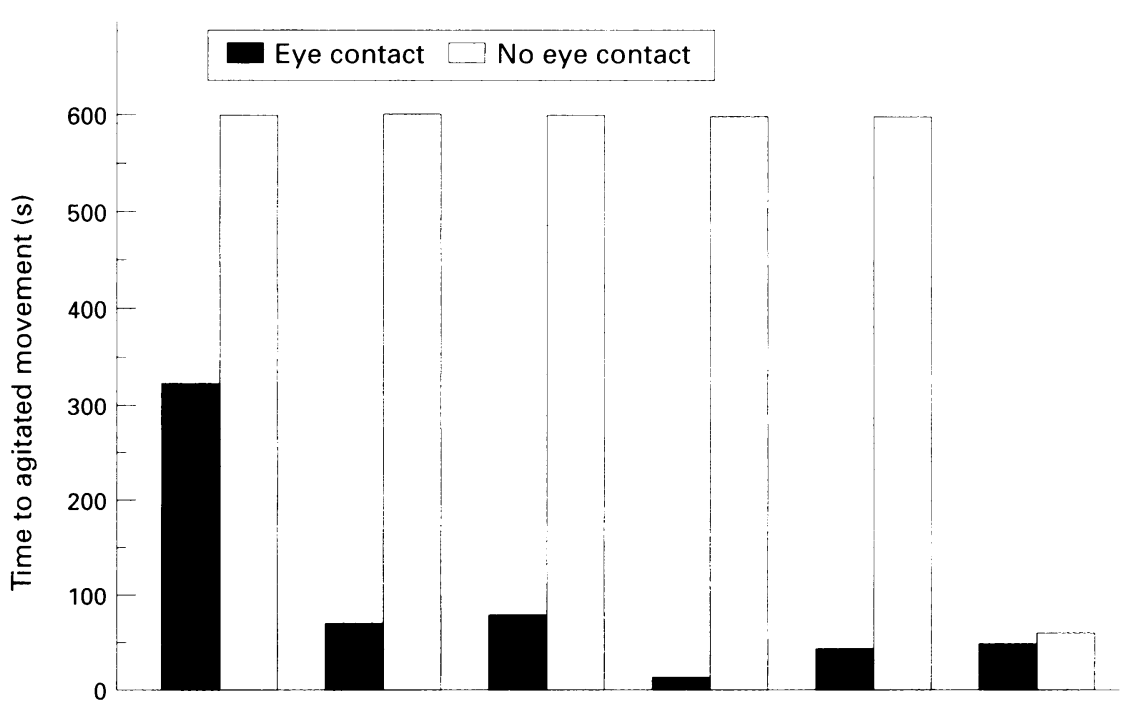

Trials $1-6$

The time taken from the experimenter approaching the patient to the first agitated movement being observed is plotted for each trial. For each of the six trials, carried out over three days, there were two conditions, one within which eye contact was made, the other with eyes averted. The trials were terminated after 10 minutes if there was no sign of agitation by this time. 